\title{
Montando o Project North Star: Um Dispositivo de Visualização de Baixo Custo Baseado em Visão Óptica Direta
}

\author{
Franklin Luiz do Nascimento Fracaro \\ Centro de Engenharias e Ciências Exatas \\ Universidade Estadual do Oeste do Paraná \\ Foz do Iguaçu, Brasil \\ frafracaro@gmail.com
}

\author{
Fabiana Frata Furlan Peres \\ Centro de Engenharias e Ciências Exatas \\ Universidade Estadual do Oeste do Paraná \\ Foz do Iguaçu, Brasil \\ fabiana.peres@unioeste.br
}

\author{
Claudio Roberto Marquetto Mauricio \\ Centro de Engenharias e Ciências Exatas \\ Universidade Estadual do Oeste do Paraná \\ Foz do Iguaçu, Brasil \\ claudio.mauricio@unioeste.br
}

\begin{abstract}
One of the major difficulties for the study of Augmented Reality using the see-through approach is the high cost of commercially available devices such as Microsoft's HoloLens or Magic Leap's Magic Leap One. Making use of the Leap Motion sensor, 3D printing, open source hardware and software, Leap Motion's startup has made available a solution for an Optical Seethrough Head Mounted Display called Project North Star. This article aims to demonstrate the process of assembling a version of this low-cost headgear and how it can become a powerful tool for the study of Augmented Reality.
\end{abstract}

Resumo-Uma das maiores dificuldades para o estudo da realidade aumentada utilizando a abordagem visão direta é o alto custo dos dispositivos disponíveis comercialmente como o HoloLens da Microsoft ou o Magic Leap One da Magic Leap. Através da união do sensor Leap Motion, impressão 3D, hardware e software de código aberto, a startup desenvolvedora do Leap Motion disponibilizou para o público uma solução de um visualizador montado na cabeça que utiliza a visão óptica direta chamado Project North Star. Este artigo tem como objetivo demonstrar o processo de montagem de uma versão deste dispositivo de baixo custo e como ele pode tornar-se uma ferramenta poderosa para o estudo da realidade aumentada.

Index Terms-Augmented reality, Optical See-Through, Head Mounted Display

\section{INTRODUÇÃO}

Azuma [1] considerou três requisitos fundamentais para definir Realidade Aumentada (RA): combinar conteúdo real e virtual; ser interativo em tempo real; e registrar em 3D. Estes requisitos determina as características de um sistema de RA, sem impor limitações quanto às tecnologias usadas [2]. Desse modo, embora os dispositivos de visualização utilizados sobre a cabeça (Head Mounted Displays), tenham sido os mais comuns durante vários anos, eles não são os únicos que viabilizam a RA [3].
Os Head Mounted Displays (HMDs) permitem que elementos virtuais sejam sobrepostos ao ambiente real com o resultado podendo ser visualizado pelo usuário [3], [4]. Estes dispositivos geralmente são do tipo visão de câmera de vídeo (video see-through) ou visão óptica direta (optical see-through) [1].

Utilizando video see-through, a imagem do mundo real visualizada pelo usuário é capturada por uma ou mais câmeras, as imagens dessas câmeras são então processadas adicionandose os objetos virtuais; o resultado é exibido no dispositivo de visualização. O optical see-through consiste em projetar através de uma superfície semitransparente, como um óculos ou viseira, os objetos virtuais ou diretamente na retina do usuário, permitindo assim a visualização dos objetos virtuais no ambiente real [5].

Embora diversas propostas de dispositivos tecnológicos tenham surgido ao longo dos anos, as limitações quanto a forma de visualização, interação, poder de processamento e principalmente custo, tem impedido a disseminação da tecnologia [6]. Nesse contexto os projetos abertos são de grande interesse visto que são tecnologias financeiramente mais acessíveis.

O Project North Star [7] é um projeto aberto com a licença GPL 3.0 [8] de um dispositivo de realidade aumentada do tipo optical see-through de baixo custo. Este artigo tem como objetivo apresentar conceitos e compartilhar a experiência do processo de montagem de uma das versões deste dispositivo, permitindo assim um melhor entendimento desta tecnologia.

\section{Fundamentação teóRica}

A popularização de smartphones e tablets mudou o foco das pesquisas, desenvolvimento e utilização da RA utilizando HMDs para RA em dispositivos móveis [9].

Atualmente a principal forma utilizada para aumentar a realidade em dispositivos móveis é o video see-through, porém 
a utilização destes aparelhos dificulta a interação do usuário além de apresentar um baixo campo de visão com o processamento da cena, resolução e contraste da imagem real sendo diretamente impactados pelo hardware disponibilizado pelo dispositivo. Estas limitações geralmente tornam um HMD do tipo video see-through baseado em dispositivos móveis inviável [6]. Os HMDs do tipo optical see-through também apresentam diversos problemas, como por exemplo, dificuldade em ocluir objetos e desenvolver-se HMDs compactos [10], porém, de acordo com Azuma [6], solucionando esses problemas, esta abordagem tem maior chance de tornar-se a principal forma utilizada em aplicações voltadas ao consumidor.

\section{A. Optical see-through}

$\mathrm{Na}$ abordagem optical see-through coloca-se combinadores ópticos na frente dos olhos do usuário como pode ser visto na Fig. 1. Esses combinadores ópticos permitem a visão direta do mundo real. Como são parcialmente reflexivos, as imagens digitais, transmitidas nos monitores acoplados ao capacete são refletidas nos combinadores, resultando na sobreposição dos objetos virtuais sobre a visão que o usuário tem do mundo real [1].

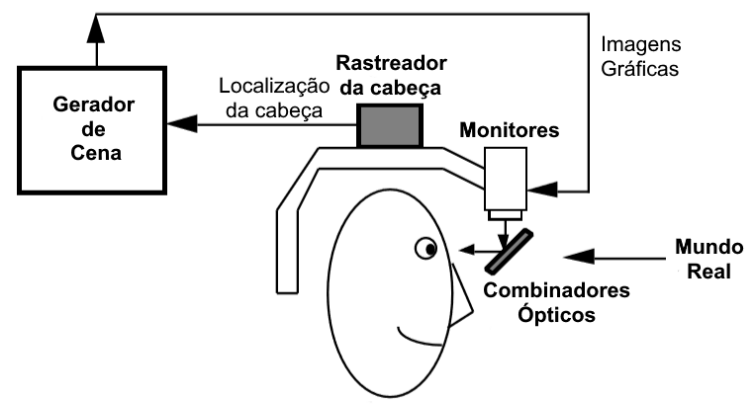

Figura 1. Esquema de um Head Mounted Display Optical See-through [1]

Para conseguir registrar e posicionar objetos virtuais no ambiente real é necessário rastrear a posição da cabeça do usuário e dos objetos presentes no mundo real. Diversas técnicas e equipamentos podem ser utilizados para alcançar este rastreamento, como por exemplo Inertial Measurement Units (IMU), que consiste de acelerômetro, giroscópio e magnetômetro, o Global Positioning System (GPS) e dispositivos como o Kinect da Microsoft e o Leap Motion Controller da Leap Motion [2], [5].

Os dados coletados por sensores são processados pela unidade responsável por gerar a cena, permitindo assim que os objetos virtuais mantenham sua posição alinhados conforme o usuário se locomove [1], [2].

\section{B. Project North Star}

O Project North Star é um projeto que foi disponibilizado pela startup desenvolvedora do Leap Motion [11] em junho de 2018. O principal objetivo deste projeto é especificar a construção de um dispositivo de visualização do tipo optical see-through acessível e barato, utilizando impressão 3D e componentes eletrônicos encontrados em lojas de equipamentos eletrônicos [12].

Em sua versão de teste antes de ser distribuído para o público, o HMD utilizava duas telas LCD de $120 \mathrm{~Hz}$ de 5.5" com resolução de $1440 \times 2560$. Cada olho visualizava conteúdo digital com aproximadamente $105^{\circ}$ verticalmente por $75^{\circ}$ horizontalmente com uma sobreposição estérea de $60 \%$, resultando em um campo de visão de $105^{\circ}$ por $105^{\circ}$ [13].

A versão mais compacta disponibilizada para o público, utiliza duas telas LCD de $120 \mathrm{~Hz}$ de 3.5 " com resolução de 1600x 1440, mantendo um campo de visão de mais de $100^{\circ}$ porém utilizando uma versão industrial do Leap Motion, não disponível para pessoas físicas, que permite o rastreamento dos gestos do usuário em um campo de $180^{\circ}$ por $180^{\circ} \mathrm{em}$ comparação ao campo de visão de $120^{\circ}$ por $150^{\circ}$ da versão comercial [12].

Com a abertura do projeto para o público através da licença GPL 3.0 surgiram outras versões [14], [15] e atualizações no projeto original [16], [17]. As novas versões apresentam propostas para adaptar componentes mecânicos e de hardware além de permitir a utilização da versão comercial do Leap Motion Controller ao invés da versão industrial.

Parte dos softwares necessários, tais como para projetar os objetos virtuais, para rastrear gestos do usuário e para projetar nas telas de LCD estão disponíveis no GitHub [18].

As partes que compõem o dispositivo especificado no Project North Star pode ser visto na Fig. 2. Ele é composto por duas telas LCD direcionadas a duas lentes com revestimento espelhado que refletem $50 \%$ e transmitem $50 \%$ da luz. Essas lentes são responsáveis por combinar as imagens virtuais. Para realizar o rastreamento e intermediar o processo de interação com os objetos virtuais e com o usuário, é utilizado o Leap Motion Controller. Estes componentes são acoplados a uma estrutura plástica impressa em uma impressora 3D.

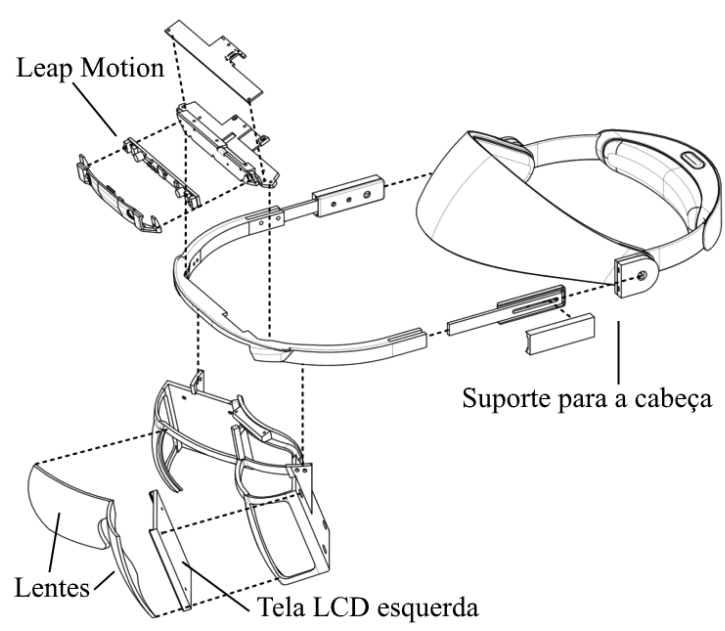

Figura 2. Esquema do Project North Star [13] 


\section{Leap Motion Controller}

O Leap Motion Controller foi lançado em 2013. Ele é uma pequena caixa de aproximadamente $7.6 \mathrm{~cm}$ de comprimento por $3 \mathrm{~cm}$ de largura e $1.3 \mathrm{~cm}$ de altura que contém duas câmeras e três LEDs infravermelhos, como pode ser visto na Fig. 3. Os LEDs são utilizados para localizar luz infravermelha com comprimentos de onda de 850 nanômetros, que está fora do espectro da luz visível. Já a câmera captura imagens em escala de cinza estéreo baseado nos dados da luz infravermelha e utiliza estes dados para calcular a posição das mãos do usuário [19].

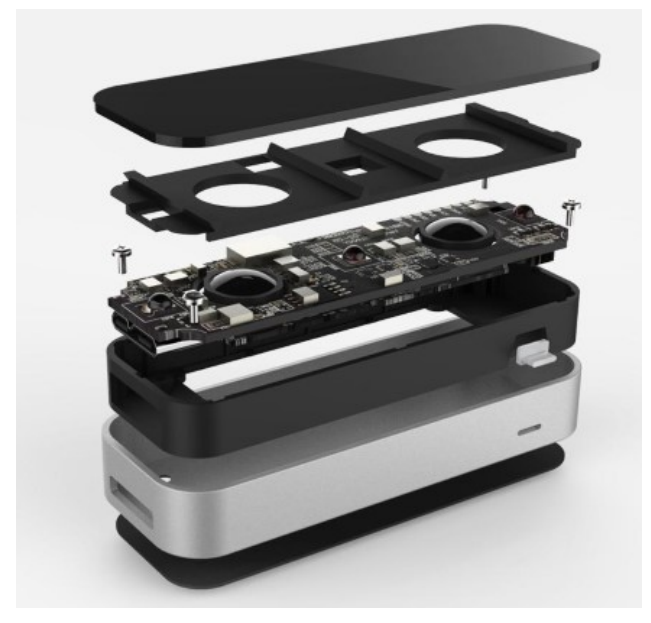

Figura 3. Leap Motion Controller [11]

O Leap Motion Controller apresenta um campo de visão vertical de $120^{\circ}$ e horizontal de $150^{\circ}$, o que resulta em um campo de visão médio de $135^{\circ}$ [20]. A distância máxima de detecção reportada oficialmente pelo fabricante é de 80 $\mathrm{cm}$, porém devido a propagação da luz dos LEDs através do espaço, esta distância poderá ser menor. A corrente que passa através da conexão USB é outro fator que restringe a capacidade de detecção pois pode afetar a intensidade dos LEDs [19].

Diversos estudos demonstram a usabilidade e robustez do Leap Motion Controller. Alguns estudos discorrem sobre a precisão de rastreamento [21], [22]; outros sobre os seus benefícios em relação a outros sistemas de rastreamento [23], programas para o reconhecimento de linguagens de sinais [24], aplicações em jogos [25], medicina [26], robótica [27], educação [20], [28], entre outros.

\section{Construindo o Project North Star}

Entre as diversas versões disponíveis do Project North Star, a versão Exiii [14] foi a escolhida para construção pois apresentou o melhor custo-benefício.

Os seguintes materiais foram adquiridos para a construção do dispositivo:

- Duas telas LCD de 3.5" HDMI para Raspberry Pi 3 Modelo B+;

- Dois cabos USB para mini USB de 2 metros;
- Um cabo HDMI para HDMI de 2 metros;

- Um cabo Mini Displayport para HDMI de 2 metros;

- Leap Motion Controller;

- 25 parafusos M2 de 6 milímetros;

- Um suporte para capacete de segurança.

O projeto requer duas portas HDMI ou uma porta HDMI e uma porta Mini Displayport para conectar as duas telas. Podese utilizar também uma porta HDMI e uma porta Thunderbolt. São necessárias também três portas USB para alimentar as duas telas e o Leap Motion Controller, podendo utilizar-se de um USB splitter para alimentar as telas, reduzindo assim a quantidade de portas USB necessárias.

A modularidade do projeto permite realizar a qualquer momento a troca das telas do Raspberry Pi por telas com taxa de atualização de $120 \mathrm{~Hz}$ que são mais caras. Embora o uso de uma tela com taxa de atualização baixa possa afetar negativamente a imersão do usuário, ela não diminui a capacidade de desenvolvimento e interação do dispositivo.

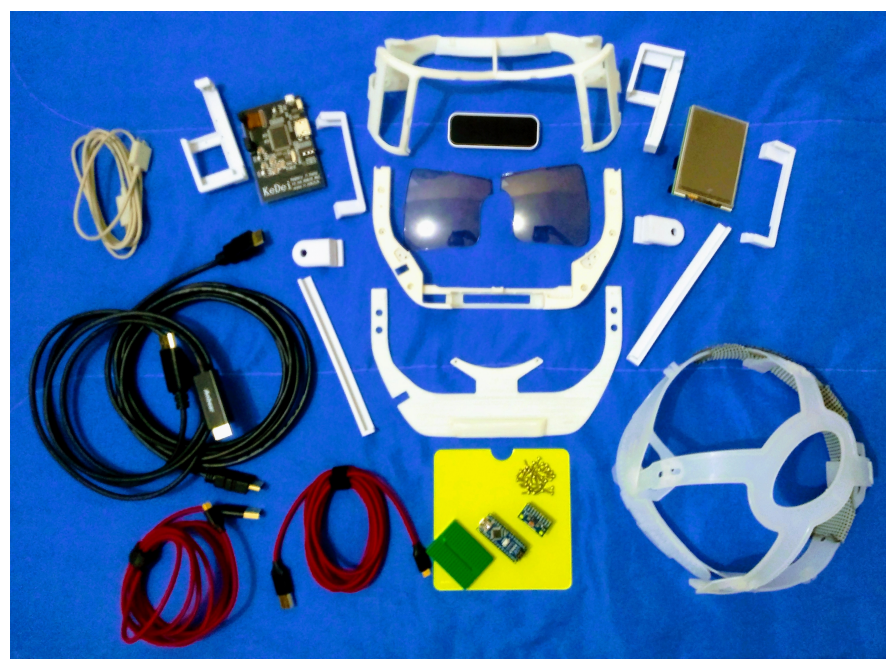

Figura 4. Componentes do Dispositivo de Visualização

Um dos maiores empecilhos encontrado durante o levantamento do projeto foi a construção das lentes. Conforme pode ser visto na Fig. 5, elas são cortadas a partir de uma estrutura elipsoide com pelo menos 2.5 milímetros de grossura. Uma alternativa seria imprimir as lentes utilizando uma impressora 3D e filamentos semitransparentes, sendo necessário realizar um processo de polimento manual até torná-las transparente aplicando-se uma película antirreflexo ao final do processo. O resultado das lentes impressas não são adequados [14], porém ajudam a diminuir os custos e permitem testar o projeto antes de comprometer-se com a compra de soluções com um custo mais elevado disponibilizadas pela comunidade do Project North Star. A solução mais adequada é a utilização de maquinário industrial [13], o que geralmente vem atrelado a um alto custo.

Devido ao projeto ser open source, a união de diversos usuários permitiu a encomenda de lentes com tratamento 


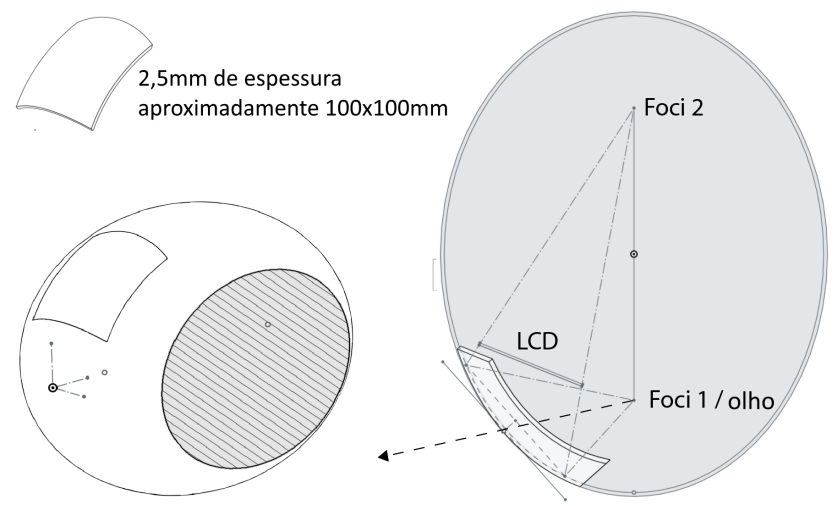

Figura 5. Lentes [13]

industrial. Elas podem ser encontradas online na Smart Prototyping [29].

Optou-se por encomendar as lentes pois devido a utilização de telas com baixa taxa de atualização, a projeção em lentes de baixa qualidade poderia prejudicar o projeto ao ponto de seu uso se tornar inviável.

O custo total dos materiais necessários para a montagem do dispositivo de visualização foi de U\$ 195,00. As peças de suporte não geraram custos, já que a Universidade Estadual do Oeste do Paraná, local onde o projeto foi desenvolvido, possui impressoras 3D incluindo uma que é fruto de um projeto para o desenvolvimento de impressoras de baixo custo [30]. No entanto, caso fosse necessário contratar esse serviço, o custo para a impressão, na presente data, varia entre U\$ 70,00 a U\$ 150,00, dependendo do modelo da impressora e tipo de filamento utilizado.

Duas impressoras diferentes foram utilizadas no processo de impressão das peças de suporte do dispositivo de visualização: CoreXY e a Prusa Mendel i3. A impressora CoreXY, foi utilizada para imprimir as três peças principais, que devido ao tamanho, exigiram uma impressora com uma área de impressão maior que 20 centímetros. Na impressora CoreXY foi utilizado o filamento do tipo PLA e filamento ABS na Prusa Mendel i3. A utilização de dois filamentos diferentes não interferiu na montagem do dispositivo ou na qualidade das peças impressas.

Com todos os materiais em mãos, foi possível montar o dispositivo sem maiores dificuldades com o resultado podendo ser visto na Fig. 7.

\section{PRÓXIMOS PASSOS}

A montagem do óculo foi um passo inicial, sendo necessário configurar e calibrar a parte do software responsável por transmitir as imagens virtuais para o dispositivo de visualização e processar os gestos capturados pelo Leap Motion controller.

Uma das expansões mais importantes para o projeto será a utilização de um IMU que inclui um acelerômetro, um giroscópio e magnetômetro acoplado a um Arduino Nano, o

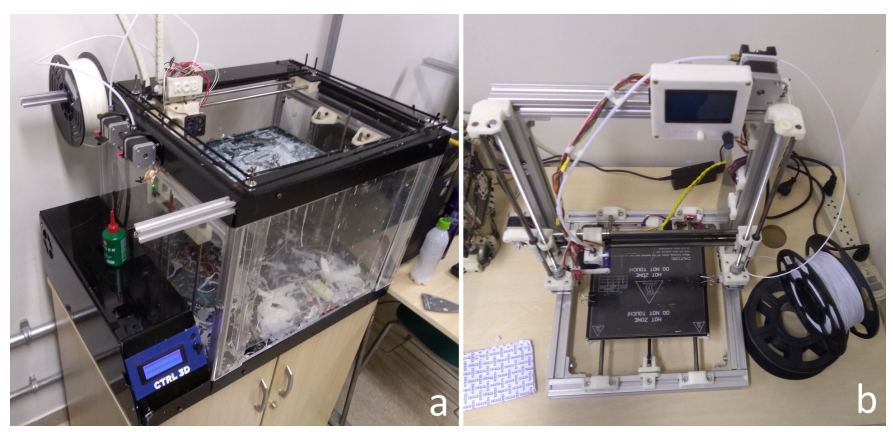

Figura 6. Impressoras Utilizadas: (a) Impressora Baseada no Modelo CoreXY e (b) Impressora Baseada no Modelo Prusa Mendel I3

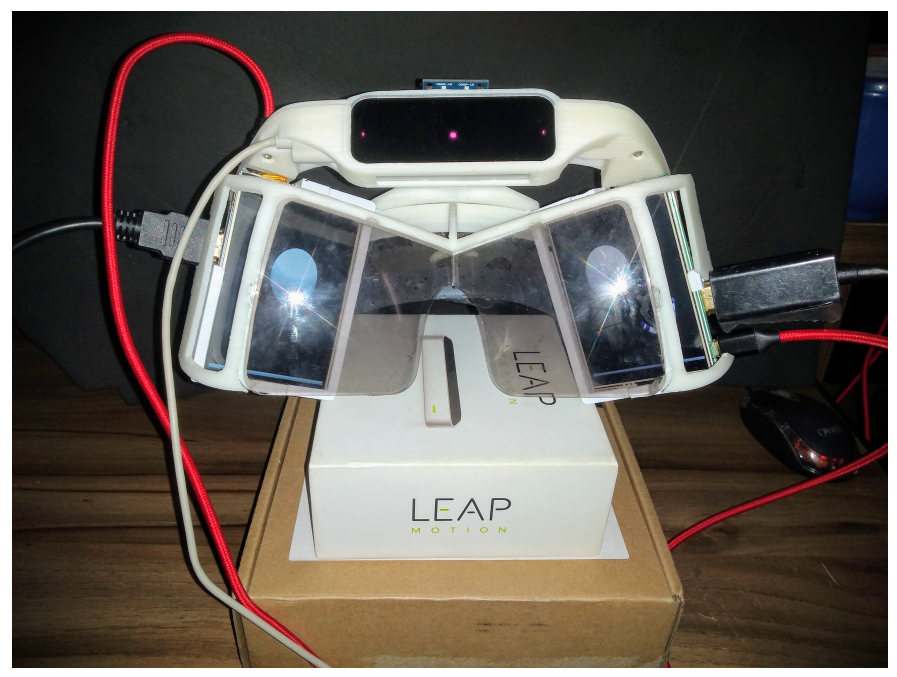

Figura 7. Dispositivo de Visualização Finalizado

que permitirá que o usuário movimente a cabeça inicialmente com 3 graus de liberdade (degrees of freedom).

Dentre as diversas rotas de pesquisa para o Project North Star está o desenvolvimento de aplicações utilizando a API do Leap Motion Controller através do Unity 3D.

A troca das telas do Raspberry $P i$ por telas com taxa de atualização de $120 \mathrm{~Hz}$ permitirá a realização de estudos comparativos entre as duas versões, analisando o impacto da taxa de atualização na experiência do usuário e em aplicativos desenvolvidos para o Project North Star.

\section{CONSIDERAÇÕES FINAIS}

Este projeto permite explorar um nível mais baixo da RA visto que muitas das funcionalidades encontradas em frameworks para dispositivos móveis acabam por abstrair diversos conceitos importantes como rastreamento, calibração e modos de exibição, similarmente, os HMDs disponíveis comercialmente atualmente tendem ao mesmo problema além do custo dificultar o acesso a estes dispositivos.

O Project North Star pode assimilar diversos dispositivos e técnicas de rastreamento porém isto acaba por levar à criação de novas versões do projeto, apesar disto a base de seu software e modo de exibição é a mesma para todos, o que 
facilita o compartilhamento de conhecimento e replicabilidade de estudos visto que existe um certo nível de padronização.

O caráter aberto do Project North Star e a flexibilidade do projeto de hardware software criam excelentes oportunidades de aplicações educacionais e considerando ainda o custo relativamente baixo para a construção do HMD, as universidades brasileiras poderiam ser beneficiadas com a sua popularização.

\section{AgRAdecimentos}

Os autores agradecem ao Prof. Antonio Marcos Massao Hachisuca, Felipe Satoru Asano e Leonardo de Jesus Queiroz por ajudarem na impressão das peças utilizadas na montagem do dispositivo de visualização.

\section{REFERÊNCIAS}

[1] R. Azuma, "A Survey of Augmented Reality", Presence: Teleoperators and Virtual Environments, vol. 6, no. 4, pp. 355-385, 1997.

[2] M. Billinghurst, A. Clark and G. Lee, "A Survey of Augmented Reality", Foundations and Trends $\AA$ in Human-Computer Interaction, vol. 8, no. 2-3, pp. 73-272, 2015.

[3] O. Bimber and R. Raskar, "Modern approaches to augmented reality", ACM SIGGRAPH 2005 Courses on - SIGGRAPH '05, 2005.

[4] J. Carmigniani, B. Furht, M. Anisetti, P. Ceravolo, E. Damiani and M Ivkovic, "Augmented reality technologies, systems and applications", Multimedia Tools and Applications, vol. 51, no. 1, pp. 341-377, 2010.

[5] R. Tori and M. S. Hounsell, Eds. Introdução a Realidade Virtual e Aumentada. Porto Alegre: Editora SBC, 2018.

[6] R. Azuma, "The road to ubiquitous consumer augmented reality systems", Human Behavior and Emerging Technologies, vol. 1, no. 1, pp. 26-32, 2019.

[7] "Project North Star - Leap Motion Developer", Leap Motion Developer, 2019. [Online]. Available: https://developer.leapmotion.com/northstar. [Accessed: 04- May2019].

[8] "The GNU General Public License v3.0- GNU Project - Free Software Foundation", Gnu.org, 2019. [Online]. Available: https://www.gnu.org/licenses/gpl-3.0.html. [Accessed: 17- Jun- 2019].

[9] A. Dey, M. Billinghurst, R. Lindeman and J. Swan II, "A Systematic Review of Usability Studies in Augmented Reality between 2005 and 2014”, 2016 IEEE International Symposium on Mixed and Augmented Reality (ISMAR-Adjunct), 2016.

[10] R. Azuma, Y. Baillot, R. Behringer, S. Feiner, S. Julier and B. MacIntyre, "Recent advances in augmented reality", IEEE Computer Graphics and Applications, vol. 21, no. 6, pp. 34-47, 2001.

[11] "Leap Motion", Leap Motion, 2019. [Online]. Available: https://www.leapmotion.com/. [Accessed: 28- May- 2019].

[12] L. Motion, "Project North Star is Now Open Source - Leap Motion Blog", Leap Motion Blog, 2019. [Online]. Available: http://blog.leapmotion.com/north-star-open-source/. [Accessed: 10- Jun2019].

[13] D. Holz and D. Holz, "Our Journey to the North Star", Leap Motion Blog, 2019. [Online]. Available: http://blog.leapmotion.com/ourjourney-to-the-north-star/. [Accessed: 24- Jun- 2019].

[14] "How to Build Project North Star Simplified Version", exiii Haptic Wearable Technology for XR, 2019. [Online]. Available: https://exiii.jp/2018/07/25/project_north_star_en/. [Accessed: 05- Mar2019].

[15] "How to Build Project North Star Simplified ver.2", exiii Haptic Wearable Technology for XR, 2019. [Online]. Available: https://exiii.jp/2018/12/07/project-north-star-simplified-v2-en/. [Accessed: 17- Jun- 2019].

[16] F. Maurer, "Project North Star: Mechanical Update 1 - Leap Motion Blog", Leap Motion Blog, 2019. [Online]. Available: http://blog.leapmotion.com/project-north-star-mechanical-update-1/. [Accessed: 03- Jul- 2019].

[17] F. Maurer, "Project North Star: Mechanical Update 3 - Leap Motion Blog", Leap Motion Blog, 2019. [Online]. Available: http://blog.leapmotion.com/project-north-star-mechanical-update-3/. [Accessed: 07- Jul- 2019].
[18] "leapmotion/ProjectNorthStar", GitHub, 2019. [Online]. Available: https://github.com/leapmotion/ProjectNorthStar. [Accessed: 03- Jul2019].

[19] A. Colgan, "How Does the Leap Motion Controller Work?", Leap Motion Blog, 2019. [Online]. Available: http://blog.leapmotion.com/hardware-to-software-how-does-the-leapmotion-controller-work/. [Accessed: 05- Jul- 2019].

[20] H. Al-Khalifa, "CHEMOTION: A gesture based chemistry virtual laboratory with leap motion", Computer Applications in Engineering Education, vol. 25, no. 6, pp. 961-976, 2017

[21] P. Valentini and E. Pezzuti, "Accuracy in fingertip tracking using Leap Motion Controller for interactive virtual applications", International Journal on Interactive Design and Manufacturing (IJIDeM), vol. 11, no. 3, pp. 641-650, 2016.

[22] F. Weichert, D. Bachmann, B. Rudak and D. Fisseler, "Analysis of the Accuracy and Robustness of the Leap Motion Controller", Sensors, vol. 13 , no. 5, pp. 6380-6393, 2013.

[23] T. Guzsvinecz, V. Szucs, and C. Sik-Lanyi, "Suitability of the Kinect Sensor and Leap Motion Controller-A Literature Review," Sensors, vol. 19 , no. 5 , p. 1072, Mar. 2019.

[24] P. Kumar, H. Gauba, P. Pratim Roy and D. Prosad Dogra, "A multimodal framework for sensor based sign language recognition", Neurocomputing, vol. 259, pp. 21-38, 2017.

[25] A. Huang, F. Huang and J. Jhu, "Unreal Interactive Puppet Game Development Using Leap Motion", Journal of Physics: Conference Series, vol. 1004, p. 012025, 2018

[26] A. Botero-Ospina, S. Duque-Vallejo, J. Ochoa-Gómez and A Hernández-Valdivieso, "Touchless control module for diagnostic images at the surgery room using the Leap Motion system and 3D Slicer Software", Revista Facultad de Ingeniería Universidad de Antioquia, no. 82, pp. 40-46, 2017.

[27] H. Jin, Q. Chen, Z. Chen, Y. Hu and J. Zhang, "Multi-LeapMotion sensor based demonstration for robotic refine tabletop object manipulation task", CAAI Transactions on Intelligence Technology, vol. 1, no. 1, pp. 104-113, 2016

[28] P. Boonbrahm, C. Kaewrat, P. Pengkaew, S. Boonbrahm and V. Meni, "Study of the Hand Anatomy Using Real Hand and Augmented Reality", International Journal of Interactive Mobile Technologies (iJIM), vol. 12, no. 7 , p. $181,2018$.

[29] "Project North Star Lens", Smart Prototyping, 2019. [Online]. Available: https://www.smart-prototyping.com/AR-VR-MR-XR/ARVR-Module/Project-North-Star-Lens. [Accessed: 09- Jul- 2019].

[30] L. Queiroz, C. Marquetto and M. Matrakas, "Construção de uma impressora 3D: Projetos abertos de baixo custo e possibilidades da aplicação na educação", The Academic Society Journal, pp. 113-118, 2019. 